\title{
Qualidade de vida de cuidadores de idosos leigos associada ao perfil sociodemográfico e situação de saúde
}

\author{
Quality of life of informal elderly caregivers associated to their sociodemographic profile and
} health conditions

Calidad de vida de los cuidadores de personas mayores no profesionales asociada al perfil sociodemográfico y condiciones de salud

Lisiane Paula Sordi Matzenbacher

\begin{abstract}
Resumo
Objetivo: analisar a qualidade de vida de cuidadores de idosos leigos associada ao perfil sociodemográfico e às suas condições de saúde. Métodos: trata-se de uma pesquisa de abordagem quantitativa, descritiva, de cunho transversal, realizada de março a maio de 2021, em que foram avaliados 22 trabalhadores leigos. Os dados foram analisados por meio de estatística descritiva simples e ANOVA. Resultados: pode-se verificar que, entre os 22 respondentes, 90,9\% são mulheres. A idade variou entre 20 e 78 anos, sendo a maioria solteira (45,5\%). Em relação ao grau de instrução, 45,5\% possuem Ensino Médio completo e 12,8\% possuem pós-graduação. Dos cuidadores, 45,5\% possuem doenças preexistentes. Foram identificadas as seguintes doenças: asma, cardiopatia, hipotireoidismo, diabetes, hipertensão arterial, osteoporose e problemas no joelho. Verifica-se que 59,1\% relatam nunca ter procurado auxílio psicológico. Observa-se que 59,01\% praticam atividades físicas. Apresenta-se diferença significativa entre os domínios físico e de relações sociais do WHOQOL-bref e entre a categoria divorciado e a categoria de cuidadores com cor de pele branca. Conclusão: esta pesquisa visa a contribuir com novos conhecimentos na área de cuidadores de idosos e auxiliar no entendimento das vulnerabilidades na qualidade de vida dessa população.
\end{abstract}

Palavras-chave: Envelhecimento; Cuidadores; Qualidade de vida; Nível saúde.

\begin{abstract}
Objective: to analyze the quality of life of informal elderly caregivers associated to their sociodemographic profile and health conditions. Methods: this is a quantitative, descriptive, cross-sectional research, carried out from March to May 2021, with 22 informal workers being evaluated. The data was analyzed using simple descriptive statistics and ANOVA. Results: it was found that among the 22 respondents, $90.9 \%$ were women, with ages ranging from 20 to 78 years, having most of them reported to be single (45.5\%). Regarding level of education, $45.5 \%$ completed high school and $12.8 \%$ had a graduate degree. Of these $45.5 \%$ of respondents had pre-existing conditions, with the following conditions being identified within this study: asthma, heart disease, hypothyroidism, diabetes, hypertension, osteoporosis, and knee
\end{abstract}


problems. Results also show that $59.1 \%$ reported never having sought psychological help, and $59.01 \%$ practiced physical activities regularly. Furthermore, final results also presented a significant difference between the physical and social relations domains of the WHOQOL-bref under the divorced and caucasian categories. Conclusion: this research aims to contribute tô new knowledge in the area of informal elderly caregivers and enable a better understanding of vulnerabilities in the quality of life of this population.

Keywords: Aging; Caregivers; Quality of life; Health status.

\section{Resumen}

Objetivo: analizar la calidad de vida de los cuidadores de ancianos legos asociada a su perfil sociodemográfico y a sus condiciones de salud. Método: se trata de una investigación con enfoque cuantitativo, descriptivo, transversal, realizada de marzo a mayo de 2021, en la que se evaluaron 22 trabajadores no profesionales. Los datos se analizaron mediante estadística descriptiva simple y ANOVA. Resultados: se puede observar que, de los 22 encuestados, el 90,9\% son mujeres. La edad osciló entre 20 y 78 años, siendo la mayoría soltera (45,5\%). En cuanto al nivel de estudios, el 45,5\% ha finalizado el bachillerato y el 12,8\% tiene un posgrado. De los cuidadores, el 45,5\% tiene enfermedades preexistentes. Se identificaron las siguientes enfermedades: asma, enfermedades cardíacas, hipotiroidismo, diabetes, hipertensión arterial, osteoporosis y problemas de rodilla. Se verifica que el 59,1\% informó no haber buscado nunca ayuda psicológica. Se observa que el 59,01\% practica actividades físicas. Existe una diferencia significativa entre los dominios de relaciones físicas y sociales del WHOQOL-bref y entre la categoría de divorciados y la categoría de cuidadores de piel blanca. Conclusión: esta investigación tiene como objetivo aportar nuevos conocimientos en el área de los cuidadores de ancianos y ayudar a comprender las vulnerabilidades en la calidad de vida de esta población.

Palabras clave: Envejecimiento; Cuidadores; Calidad de vida; Estado de salud.

\section{Introdução}

A atual transição demográfica aponta que pessoas de todo o mundo estão vivendo mais e é possível que a maioria delas chegue a viver 60 anos ou mais. Espera-se, em 2050, que a população na faixa etária de 60 anos chegue a dois bilhões, em contrapartida, em 2015, os dados apontavam 900 milhões de idosos no mundo (Organização Pan-Americana de Saúde, 2018).

Quando se trata dos idosos, no Brasil, esse aumento segue os passos da população mundial e a população com 60 anos ou mais tem tendência a aumentar. É o que traz os dados do IBGE, que apontam que as pessoas com 65 anos ou mais já representam 10\% da população brasileira e, até 2060, os idosos devem ser 25,5\% do total da população do país (Brasil, 2019).

Uma vida mais longa traz oportunidades para as pessoas idosas, suas famílias e para toda a sociedade. Esses anos a mais de vida geram a possibilidade de buscar novas atividades, como educação, carreira ou viver novas paixões. As pessoas idosas contribuem de muitas formas para suas famílias e comunidades. Essas oportunidades estão relacionadas com um fator primordial: a saúde (Organização Pan-Americana de Saúde, 2018).

Mesmo com a criação de políticas públicas que favorecem o idoso, essas ainda não são suficientes para o atendimento dessa população no país. Em busca de um envelhecimento saudável, mostram-se necessários novas formas de atenção à população e um aprofundamento de trabalhos acadêmicos na área, dando a devida atenção a essa população, além de uma maior aplicabilidade de políticas já existentes (Sousa et al., 2020).

Com o aumento da população idosa, é necessário ter atenção aos trabalhadores que cuidam de idosos e como está a sua qualidade de vida. A sobrecarga deve ser vista como um conceito multidimensional que abrange muitas variáveis (Cardoso et al., 2020). Em uma pesquisa realizada com 86 cuidadores, os dados mostraram que 55,8\% apresentavam dificuldades nos cuidados com os idosos como: fazer esforço físico (20,9\%) na transferência do idoso e com os problemas de saúde que geram dor ou desconforto na pessoa que necessita de assistência (Jesus et al., 2018).

Resultados na literatura apontaram que a carga de trabalho desses cuidadores está baseada em estressores como: a idade; a escolaridade; o tempo que dedicam ao idoso; o grau de parentesco; a convivência e o uso de renda própria para custear os cuidados, demonstrando a necessidade de mais estudos sobre o tema (Aires et al., 2020). Há evidências da necessidade de conhecer o perfil desses cuidadores, a sobrecarga e as consequências para a vida tanto do cuidador quanto do idoso (Jesus et al., 2018). 
O enfermeiro, como um profissional voltado ao cuidado eficiente, deve estar atento a todo o processo de cuidado, considerando aquele que presta o cuidado e quem o recebe. Os profissionais de Enfermagem têm atuação direta no papel de educação permanente dos cuidadores de idosos. Assim, esta pesquisa poderá auxiliar os profissionais de Enfermagem no entendimento das demandas dos cuidadores de idosos.

Quando se fala em cuidado ao idoso, deve-se considerar que a qualidade de vida do cuidador reflete nos cuidados prestados. Assim, há a necessidade de estudos que reforcem e analisem como está a qualidade de vida desses trabalhadores, sejam eles familiares ou trabalhadores formais. Com isso, este estudo visa a responder à seguinte pergunta: o perfil sociodemográfico e as condições de saúde de cuidadores de idosos leigos estão associados à qualidade de vida? O objetivo deste estudo é analisar a qualidade de vida de cuidadores de idosos leigos associada ao perfil sociodemográfico e às suas condições de saúde.

\section{Métodos}

Trata-se de uma pesquisa de abordagem quantitativa realizada de março a maio de 2021. A pesquisa quantitativa atua sobre uma problemática humana ou social, baseada em uma teoria e sua composição, e as variáveis são quantificadas em números e têm como objetivo verificar se as generalizações esperadas em teorias se mantêm ou não (Knechtel, 2014).

O cenário de investigação do estudo foi um projeto de extensão denominado "Qualidade de vida em cuidadores leigos: uma reflexão em relação ao envelhecimento", que é uma atividade voltada para cuidadores de idosos. Essa atividade é realizada pela Escola de Enfermagem da Universidade Federal do Rio Grande do Sul (UFRGS). Devido ao atual cenário da pandemia da COVID-19, os participantes recebem, semanalmente, materiais para estudo e debatem via aplicativos de mensagens.

A população estudada foi composta por cuidadores leigos de idosos, sendo ou não familiares do idoso, que participaram do projeto de extensão, totalizando 22 pessoas que cuidam de idosos em diferentes locais. Foram realizados contato telefônico e contato por via de aplicativos de mensagens para a aplicação dos questionários. Os critérios de inclusão foram cuidadores de idosos, de ambos os sexos, independentemente de idade. Os critérios de exclusão da pesquisa são pessoas não encontradas ou em férias no momento da pesquisa.

Os participantes da pesquisa foram convidados a responder a dois instrumentos dentro do período estudado, sendo oferecido um Termo de Compromisso Livre e Esclarecido (TCLE) àqueles que concordaram em participar do estudo. O primeiro instrumento trata-se de um questionário semiestruturado desenvolvido para a coleta de dados sociodemográficos e informações sobre a situação de saúde. O segundo instrumento é o World Health Quality of Life, conhecido como WHOQOL-bref, voltado para a avaliação de qualidade de vida.

A escala de medida utilizada no instrumento WHOQOL é a de likert de cinco pontos. Para a coleta de dados da pesquisa, em virtude do cenário atual da pandemia da COVID-19, os questionários foram aplicados via Google Forms®.

Foi utilizado o programa R (R Core Team, 2021) para calcular a estatística descritiva básica como a porcentagem de distribuição, média, desvio-padrão dos respondentes, os dados demográficos e escores dos domínios de WHOQOL-BREF. Para a análise da consistência interna, foi realizado o teste Alfa de Cronbach, considerando-se acima de 0.7 como aceitável (Cronbach, 1951). A comparação entre os grupos de cuidadores foi realizada considerando as seguintes variáveis relacionadas aos dados sociodemográficos: idade, gênero, estado civil, escolaridade, cor, religião, renda e doenças. Também foram avaliadas as condições de saúde com base nas doenças pré-existentes, realização de exercícios físicos e procura de assistência psicológica. Para as comparações entre os dados sociodemográficos e os domínios, foram realizados o teste de normalidade, a análise de variância e o teste de Tukey, sendo considerado significativo $p<0,05$. 
Os aspectos éticos presentes no estudo respeitam a Resolução n ${ }^{\circ}$ 466, de 12 de dezembro de 2012, que trata dos estudos realizados com seres humanos e preza pela proteção e dignidade dos mesmos durante as pesquisas (Brasil, 2012). O projeto foi aprovado na plataforma Brasil sob o Parecer de nº 4.663.281.

Devem-se descrever, de forma clara e sem prolixidade, as fontes de dados, a população estudada, a amostragem, os critérios de seleção, os procedimentos analíticos e as questões éticas relacionadas à aprovação do estudo por comitê de ética em pesquisa (pesquisa com seres humanos e animais) ou autorização institucional (levantamento de dados onde não há pesquisa direta com seres humanos ou animais).

\section{Resultados e Discussão}

A amostra foi composta por 90,9\% (20) de mulheres e 9,1\% (2) de homens. Podemos observar na Tabela 1, que a idade variou entre 20 e 78 anos, sendo a maioria solteira (45,5\%). Em relação ao grau de instrução, 45,5\% possuem Ensino Médio completo e observa-se que 12,8\% possuem pós-graduação. Em relação à cor, a maioria dos respondentes declarou-se branca $(86,4 \%)$. Em relação à renda, 22,7\% (5) dos respondentes disseram não estar trabalhando no momento, uma pessoa declarou-se autônoma e não informou a renda bruta. A renda flutuou entre $\mathrm{R} \$ 1.200,00$ a $\mathrm{R} \$ 15.0000,00$, com uma média de $\mathrm{R} \$ 2.229,00$ (cerca de dois salários-mínimos), sendo mais alta entre as pessoas que apresentavam pós-graduação. O peso médio dos cuidadores foi de 70,8 kg com desvio-padrão de $13,3 \mathrm{~kg}$ (sendo que a média feminina foi de $71 \mathrm{~kg}$ e a masculina de $69 \mathrm{~kg}$ ). A altura média foi $1,61 \mathrm{~m}$ com desvio-padrão 0,07 .

Quando questionados se já procuraram assistência psicológica, 59,1\% relatam não ter procurado auxílio. Em relação a doenças, $54,5 \%$ dos cuidadores não possuem doenças preexistentes. Dentre os $45,5 \%$ que apresentaram doenças, foram identificadas, pelos cuidadores, as seguintes doenças: asma, cardiopatia, hipotireoidismo, diabetes, hipertensão arterial, osteoporose e problemas no joelho. Sobre apresentar religião, 86,4\% dos respondentes afirmaram ter, sendo relatadas as seguintes religiões: católica, espírita, evangélica, batuqueira e candomblecista, budista, luterana e testemunha de Jeová. 
Tabela 1 - Perfil sociodemográfico de cuidadores de idosos, Porto Alegre/RS, Brasil 2021.

\begin{tabular}{|c|c|c|}
\hline Características & \multicolumn{2}{|c|}{$\%$} \\
\hline \multicolumn{3}{|l|}{ Gênero } \\
\hline Mulheres & 20 & 90,9 \\
\hline Homens & 2 & 9,1 \\
\hline \multicolumn{3}{|l|}{ Estado Civil } \\
\hline Casado(a) & 5 & 22,7 \\
\hline Solteiro(a) & 4 & 45,5 \\
\hline Divorciado(a) & 10 & 18,1 \\
\hline Viúvo(a) & 3 & 13,7 \\
\hline \multicolumn{3}{|l|}{ Escolaridade } \\
\hline Ensino incompleto & 1 & 4,5 \\
\hline $\begin{array}{l}\text { Ensino Fundamental } \\
\text { completo }\end{array}$ & 1 & 4,5 \\
\hline Ensino Médio completo & 10 & 45,5 \\
\hline Ensino Superior completo & 6 & 27,3 \\
\hline Pós-graduação & 4 & 18,2 \\
\hline \multicolumn{3}{|l|}{ Cor } \\
\hline Branca & 19 & 86,4 \\
\hline Parda & 1 & 4,5 \\
\hline Preta & 2 & 9,1 \\
\hline \multicolumn{3}{|l|}{ Psicólogo } \\
\hline $\operatorname{Sim}$ & 9 & 40,9 \\
\hline Não & 13 & 59,1 \\
\hline \multicolumn{3}{|l|}{ Doença } \\
\hline Sim & 10 & 45,5 \\
\hline Não & 12 & 54,5 \\
\hline \multicolumn{3}{|l|}{ Religião } \\
\hline Sim & 19 & 86,4 \\
\hline Não & 3 & 13,6 \\
\hline
\end{tabular}

Fonte: Autores.

A literatura refere que as doenças prevalentes em cuidadores de idosos são a hipertensão arterial sistêmica, as doenças do sistema locomotor e o Diabetes Mellitus. Outras doenças também foram encontradas nos resultados do artigo e estão pouco listadas na literatura voltada a cuidadores de idosos (Brandão et al., 2017). Um estudo realizado com idosos que prestam cuidado a outros idosos relatou que os aspectos da saúde física (sinais e sintomas, doenças crônicas e percepção do agravamento da saúde), juntamente com a sobrecarga, influenciam a qualidade de vida desse tipo de cuidador (Flesh, 2019).

Já os dados relacionados com a procura de assistência psicológica apresentam-se um pouco maiores (40,9\%) que os encontrados em um trabalho realizado com cuidadores de idosos com demência em que cerca de 34,0\% dos entrevistados afirmaram fazer psicoterapia, enquanto 65,7\% afirmaram nunca ter feito algum tipo de acompanhamento psicoterápico (Queiroz et al., 2021). A terapia promove intervenções que podem remediar e reduzir o sofrimento de acordo com cada cuidador (Paulo et al., 2019).

Em relação às atividades físicas, 40,9\% dos respondentes não praticam e 59,01\% relataram praticar atividades físicas, pelo menos, uma vez por semana regularmente, demonstrando que a prática de exercícios físicos foi mais presente que na pesquisa realizada por Muniz et al. (2016), em que a não realização de atividades físicas regulares somava 88,3\% entre os cuidadores de idosos. Aranda (2018) realizou um estudo com idosos em que a prática de atividade física interferiu diretamente na qualidade de vida, independência funcional, função física (vitalidade), autoestima, funcionamento cognitivo, bem-estar, saúde mental, otimismo, envelhecimento saudável e integração social. 
Neste estudo, a consistência interna foi de 0.855 , sendo considerada substancial ou boa (Landis \& Kock, 1977). Em um nível de significância de 5\%, não se rejeita a normalidade das respostas, portanto, podem-se fazer testes ANOVA para ver a influência das variáveis sociodemográficas e de comorbidades nos escores.

Em relação ao estado civil e aos domínios, foi encontrada uma diferença significativa na categoria divorciado e entre os domínios físico e de relações sociais ( $\mathrm{p}=0,0274$ ), o que pode ser observado na tabela 2 . Um estudo realizado na China com cuidadores de idosos com doenças psiquiátricas associou o estado civil divorciado com a baixa qualidade de vida dos cuidadores de idosos (Neong \& Rashid, 2018). Hajebi et al. (2019) observaram que isso se deve ao fato de os cuidadores de idosos divorciados apresentarem uma sobrecarga maior em relação aos casados. É possível inferir que essa realidade se deve à falta de suporte social para as atividades do dia a dia, que é evidenciada dentro dos domínios físico e de relações sociais.

Tabela 2 - Análise de variância entre os domínios do WHOQOL-bref e o estado civil, Porto Alegre/RS, Brasil, 2021. - Intervalo de Confiança 95\%.

\begin{tabular}{|c|c|c|c|c|c|c|}
\hline Domínios & & Diferenças das médias & $\begin{array}{c}\text { Erro } \\
\text { Padrão }\end{array}$ & p-valor & Limite Inferior & Limite Superior \\
\hline \multicolumn{7}{|l|}{ Solteiro(a) } \\
\hline \multirow[t]{3}{*}{ Físico } & Psicológico & 11,4 & 7,02 & 0,3779 & $-7,5$ & 30,3 \\
\hline & Relações Sociais & 12,1 & 7,02 & 0,3262 & $-6,8$ & 31 \\
\hline & Ambiental & 13,8 & 7,02 & 0,2193 & $-5,1$ & 32,7 \\
\hline \multirow[t]{2}{*}{ Psicológico } & Relações Sociais & 0,7 & 7,02 & 0,9996 & $-18,2$ & 19,6 \\
\hline & Ambiental & 2,4 & 7,02 & 0,986 & $-16,5$ & 21,3 \\
\hline Relações Sociais & Ambiental & 1,7 & 7,02 & 0,9949 & $-17,2$ & 20,6 \\
\hline \multicolumn{7}{|l|}{ Divorciado(a) } \\
\hline \multirow[t]{3}{*}{ Físico } & Psicológico & 3,25 & 7,54 & 0,972 & $-19,14$ & 25,6 \\
\hline & Relações Sociais & 25 & 7,54 & $0,0274 *$ & 2,61 & 47,4 \\
\hline & Ambiental & 14 & 7,54 & 0,2959 & $-8,39$ & 36,4 \\
\hline \multirow[t]{2}{*}{ Psicológico } & Relações Sociais & 21,75 & 7,54 & 0,0578 & $-8,64$ & 44,1 \\
\hline & Ambiental & 10,75 & 7,54 & 0,5082 & $-11,64$ & 33,1 \\
\hline $\begin{array}{l}\text { Relações Sociais } \\
\text { Casado(a) }\end{array}$ & Ambiental & -11 & 7,54 & 0,4898 & $-33,39$ & 11,4 \\
\hline \multirow[t]{3}{*}{ Físico } & Psicológico & $-2,6$ & 11,2 & 0,9954 & $-34,7$ & 29,5 \\
\hline & Relações Sociais & 2,6 & 11,2 & 0,9954 & $-29,5$ & 34,7 \\
\hline & Ambiental & $-1,4$ & 11,2 & 0,9993 & $-33,5$ & 30,7 \\
\hline \multirow[t]{2}{*}{ Psicológico } & Relações Sociais & 5,2 & 11,2 & 0,966 & $-26,9$ & 37,3 \\
\hline & Ambiental & 1,2 & 11,2 & 0,9995 & $-30,9$ & 33,3 \\
\hline $\begin{array}{l}\text { Relações Sociais } \\
\text { Viúvo(a) }\end{array}$ & Ambiental & -4 & 11,2 & 0,9839 & $-36,1$ & 28,1 \\
\hline \multirow[t]{3}{*}{ Físico } & Psicológico & -10 & 9,27 & 0,711 & $-39,68$ & 19,7 \\
\hline & Relações Sociais & 15 & 9,27 & 0,421 & $-14,68$ & 44,7 \\
\hline & Ambiental & -2 & 9,27 & 0,9962 & $-31,68$ & 27,7 \\
\hline \multirow[t]{2}{*}{ Psicológico } & Relações Sociais & 25 & 9,27 & 0,1019 & $-4,68$ & 54,7 \\
\hline & Ambiental & 8 & 9,27 & 0,8233 & $-21,68$ & 37,7 \\
\hline Relações Sociais & Ambiental & -17 & 9,27 & 0,3256 & $-46,68$ & 12,7 \\
\hline
\end{tabular}

Fonte: Autores.

$\mathrm{Na}$ Tabela 3, foram analisadas as diferenças de cor com as dimensões na cor branca. Foi encontrada uma diferença significativa entre a dimensão física e a das relações sociais ( $\mathrm{p}=0,0339)$, enquanto, nas outras categorias, não foi encontrada diferença significativa ( $p>0,05)$. Estudo realizado com cuidadores de pessoas com demência relatou que, em cuidadores brancos, a satisfação com o suporte social, a ajuda doméstica e a participação em grupos de apoio demonstraram resultados significativos dentro de aspectos positivos de cuidado (Cho et al., 2016). Cerca de 18\% dos cuidadores brancos prestam cuidados diariamente em comparação com $21 \%$ dos asiáticos, $24 \%$ dos negros e cerca de $30 \%$ dos mexicanos-americanos e outros cuidadores latinos 
(Rote \& Moon, 2018). Esses dados apresentados corroboram os domínios do WHOQOL-bref referentes às atividades do dia a dia e ao suporte social.

Tabela 3 - Análise de variância entre os domínios do WHOQOL-bref e cor, Porto Alegre/RS, Brasil, 2021. Intervalo de Confiança $95 \%$.

\begin{tabular}{|c|c|c|c|c|c|c|}
\hline Domínios & & Diferenças das médias & $\begin{array}{c}\text { Erro } \\
\text { Padrão }\end{array}$ & p-valor & Limite Inferior & Limite Superior \\
\hline \multicolumn{7}{|l|}{ Branco(a) } \\
\hline \multirow[t]{3}{*}{ Físico } & Psicológico & 2,79 & 5,15 & 0,9488 & -10.784 & 16,36 \\
\hline & Relações Sociais & 14,37 & 5,16 & $0,0339 *$ & 0,795 & 27,94 \\
\hline & Ambiental & 5,32 & 5,16 & 0,7325 & -8.257 & 18,89 \\
\hline \multirow[t]{2}{*}{ Psicológico } & Relações Sociais & 11,58 & 5,16 & 0,1214 & -1.994 & 25,15 \\
\hline & Ambiental & 2,53 & 5,16 & 0,9612 & -11.047 & 16,1 \\
\hline Relações Sociais & Ambiental & $-9,05$ & 5,16 & 0,3039 & -22.626 & 4,52 \\
\hline Pardo(a) & & & & & & \\
\hline \multirow[t]{3}{*}{ Físico } & Psicológico & 13 & $\mathrm{NaN}$ & $\mathrm{NaN}$ & $\mathrm{NaN}$ & $\mathrm{NaN}$ \\
\hline & Relações Sociais & 19 & $\mathrm{NaN}$ & $\mathrm{NaN}$ & $\mathrm{NaN}$ & $\mathrm{NaN}$ \\
\hline & Ambiental & 44 & $\mathrm{NaN}$ & $\mathrm{NaN}$ & $\mathrm{NaN}$ & $\mathrm{NaN}$ \\
\hline \multirow[t]{2}{*}{ Psicológico } & Relações Sociais & 6 & $\mathrm{NaN}$ & $\mathrm{NaN}$ & $\mathrm{NaN}$ & $\mathrm{NaN}$ \\
\hline & Ambiental & 31 & $\mathrm{NaN}$ & $\mathrm{NaN}$ & $\mathrm{NaN}$ & $\mathrm{NaN}$ \\
\hline Relações Sociais & Ambiental & 25 & $\mathrm{NaN}$ & $\mathrm{NaN}$ & $\mathrm{NaN}$ & $\mathrm{NaN}$ \\
\hline $\operatorname{Preto}(\mathbf{a})$ & & & & & & \\
\hline \multirow[t]{3}{*}{ Físico } & Psicológico & 9 & 22,8 & 0,9764 & $-83,7$ & 101,7 \\
\hline & Relações Sociais & $-6,5$ & 22,8 & 0,9907 & $-99,2$ & 86,2 \\
\hline & Ambiental & 18 & 22,8 & 0,8559 & $-74,7$ & 110,7 \\
\hline \multirow[t]{2}{*}{ Psicológico } & Relações Sociais & $-15,5$ & 22,8 & 0,8995 & $-108,2$ & 77,2 \\
\hline & Ambiental & 9 & 22,8 & 0,9764 & $-83,7$ & 101,7 \\
\hline Relações Sociais & Ambiental & 24,5 & 22,8 & 0,7208 & $-68,2$ & 117,2 \\
\hline
\end{tabular}

Fonte: Autores.

Em relação à religião como um fator que afeta os domínios, foi encontrada uma diferença de 0,0557 entre o domínio físico e o das relações sociais, enquanto os demais domínios apresentaram p>0,05 (Tabela IV). Apesar de não apresentar um valor significativo nesses domínios, a literatura traz a religião como um processo de suporte à rotina e aos cuidados que são realizados sozinhos com o idoso (Silva et al., 2019). Em um trabalho realizado com cuidadores informais de idosos hospitalizados, foi possível concluir que esses cuidadores utilizam crenças religiosas para enfrentar o estresse recorrente do cuidado (Vitorino et al., 2018). 
Tabela4 - Análise de variância entre a religião e os domínios do questionário, Porto Alegre/RS, Brasil, 2021.

\begin{tabular}{|c|c|c|c|c|c|c|}
\hline \multicolumn{2}{|l|}{ Domínios } & Diferenças das médias & Erro Padrão & p-valor & Limite Inferior & Limite Superior \\
\hline \multicolumn{7}{|c|}{ Tem religião } \\
\hline \multirow[t]{2}{*}{ Físico } & Psicológico & 4,11 & 5,28 & 0,8651 & -9.811 & 18,02 \\
\hline & Relações Sociais & 13,68 & 5,29 & 0,0557 & $-0,232$ & 27,60 \\
\hline \multirow{3}{*}{ Psicológico } & Ambiental & 6,58 & 5,29 & 0,6016 & -7.337 & 20,49 \\
\hline & Relações Sociais & 9,58 & 5,29 & 0,2769 & -4.337 & 23,49 \\
\hline & Ambiental & 2,47 & 5,29 & 0,9659 & -11.442 & 16,39 \\
\hline \multirow{2}{*}{\multicolumn{2}{|c|}{$\begin{array}{c}\text { Relações Sociais Ambiental } \\
\text { Não tem religião }\end{array}$}} & $-7,11$ & 5,29 & 0,539 & -21.021 & 6,81 \\
\hline & & & & & & \\
\hline \multirow[t]{3}{*}{ Físico } & Psicológico & 2,00 & 15 & 0,9991 & $-46,1$ & 50,10 \\
\hline & Relações Sociais & 6,33 & 15 & 0,9732 & $-41,8$ & 54,50 \\
\hline & Ambiental & 18,67 & 15 & 0,62 & $-29,5$ & 66,80 \\
\hline \multirow[t]{2}{*}{ Psicológico } & Relações Sociais & 4,33 & 15 & 0,991 & $-43,8$ & 52,50 \\
\hline & Ambiental & 16,67 & 15 & 0,6944 & $-31,5$ & 64,80 \\
\hline Relações Sociais & Ambiental & 12,33 & 15 & 0,8433 & $-35,8$ & 60,50 \\
\hline
\end{tabular}

Fonte: Autores.

\section{Conclusão}

Constatou-se a fragilidade de suporte aos cuidadores de idosos que não apresentam companheiros. O cuidador, na maioria do sexo feminino, vê-se sozinho nos cuidados e isso gera sobrecarga, aumentando a probabilidade de aparecimento de futuras doenças. Quando se trata de qualidade de vida, observaram-se a cor de pele branca como um fator relacionado com a satisfação com o cuidado e a rotina de cuidados menos branda, interferindo, positivamente, nas atividades do dia a dia quando comparadas às das pessoas com outras cores de pele.

É possível inferir que o sistema de saúde público brasileiro não está preparado para atender às demandas do crescimento populacional de idosos e, respectivamente, dos seus cuidadores. Os achados apontam a necessidade de políticas públicas que promovam o suporte a essa população, visando à redução da sobrecarga relacionada às atividades inerentes à prestação de cuidados.

Apesar da participação em grupos de suporte, ainda se apresentam fragilidades na qualidade de vida dos cuidadores de idosos. Este estudo pode contribuir na identificação de populações com vulnerabilidades e no planejamento do cuidado delas. Os profissionais de Enfermagem têm atuação direta no papel de educação permanente dos cuidadores de idosos.

\section{Referências}

Aires, M., Fuhrmann, A. C., Mocellin, D., Dal Pizzol, F. L. F., Sponchiado, L. F., Marchezan, C. R., Bierhals, C. C. B. K., Day, C. B., Santos, N. O., \& Paskulin, L. M. G. (2020). Burden of informal caregivers of dependent elderlies in the community in small cities. Revista Gaúcha de Enfermagem, 41 (Spe), e20190156.

Aranda, R. M. (2018). Actividad física y calidad de vida en el adulto mayor. Una revisión narrativa. Habanera Journal of Medical Sciences, 17(5), 813-825.

Brandão, G. C. G., Dias, A. M., Saad, D., Cartaxo, R. M. S., Silveira, M. J. O., \& Silva, C. M. (2017). Perfil da saúde dos cuidadores de idosos: uma revisão integrativa. RSC online, 6(1), 96-113.

Brasil. (2019). Confederação Nacional de Municípios. Crescimento da população idosa reforça necessidade de ações destinadas ao público. Brasília: CNM.

Brasil. (2012). Resolução $n^{\circ} 466$, de 12 de dezembro de 2012. Ministério da Saúde.

Cardoso, S. M. M., Rodrigues, A. P., Sperling, S. G., Machado, N. C. B., Rocha, L. S., Machado, M. T. K., \& Fernandes, R. F. M. (2020). Cuidadores de idosos em estratégias de saúde da família: o estresse destes indivíduos. Revista Eletrônica Acervo Saúde, 49(Suppl 49 ), e3179.

Cho, J., Ory, M. G., \& Stevens, A. B. (2016). Socioecological factors and positive aspects of caregiving: findings from the REACH II intervention. Aging \& Mental Health, 20(11), 1190-1201.

Cronbach, L. J. (1951). Coefficient alpha and the internal structure of tests. Psychometrika, 16, 297-334. 
Flesh, L. D. (2019). Factors associated with the quality of life of elderly caregivers of other elderly persons. Revista Brasileira de Geriatria e Gerontologia, 22(3), e180155.

Hajebi, A., Naserbakht, M., \& Minoletti, A. (2019). Burden experienced by caregivers of schizophrenia patients and its related factors. Medical Journal of Islamic Republic of Iran, 33, 54

Jesus, I. T. M., Orlandi, A. A. S., \& Zazzetta, M. S. (2018). Burden, profile and care: caregivers of socially vulnerable elderly persons. Revista Brasileira de Geriatria e Gerontologia, 21(2), 194-204.

Knechtel, M. R. (2014). Metodologia da pesquisa em educação: uma abordagem teórico-prática dialogada. Intersaberes, 11(2), 531-534.

Landis, J. R., \& Koch, G. G. (1977). The measurement of observer agreement for categorical data. Biometrics, 33(1), 159-174.

Muniz, E. A., Freitas, C. A. S. L., Oliveira, E. N., \& Lacerda, M. R. (2016). Grau de sobrecarga dos cuidadores de idosos atendidos em domicílio pela Estratégia Saúde da Família. Saúde em Debate, 40(110), 172-182.

Neong, S. C., \& Rashid, A. (2018). Quality of life of caregivers of patients with psychiatric illness in Penang. Journal Clinical Psychiatry and Cognitive Psychology, 2(1), 12-18.

Organização Pan-Americana de Saúde. (2018). Envelhecimento e Saúde. OPAS.

Paulo, D. L.V., Deienno, S. C. P., \& Oliveira, A. (2019). Cuidadores de Idosos. A importância do atendimento psicológico. Revista Longeviver, 3, 65-68.

Queiroz, R. S., Camacho, A. C. L. F., Gurgel, J. L., Assis, C. R. C., Santos, L. M., \& Santos, M. L. S. C. (2018). Perfil sociodemográfico e qualidade de vida de cuidadores de idosos com demência. Revista Brasileira de Geriatria e Gerontologia, 21(2), 210-219.

R Core Team. (2021). R: a language and environment for statistical computing. R Foundation for Statistical Computing.

Rote, S. M., \& Moon, H. (2018). Racial/ethnic differences in caregiving frequency: does immigrant status matter? The Journals of Gerontology, 73(6), 10881098.

Silva, R. M., Borges, R. T., Gonçalves, J. L., Bezerra, I. C., Vieira, L. J. E. S., \& Saintrain, M. V. L. (2019). A religiosidade no amparo ao cuidador de idoso dependente. Atas - Investigação Qualitativa em Saúde, 2, 1778-1783.

Sousa, M. C., Barroso, I. L. D., Viana, J. A., Ribeiro, J. N., Lima, L. N. F., Vanccin, P. D. A., Silva, V. G. P., \& Nascimento, W. C. (2020). O envelhecimento da população: aspectos do Brasil e do mundo, sob o olhar da literatura. Brazilian Journal of Developmrnt, 6(8), 61871-61877.

Vitorino, L. M., Marins, L. S., Lucchetti, A. L. G., Santos, A. E. O., Cruz, J. P., Cortez, P. J. O., \& Lucchetti, G. (2018). Spiritual/religious coping and depressive symptoms in informal caregivers of hospitalized older adults. Geriatric Nursing, 39(1), 48-53. 